\title{
On the discrepancy estimate of normal numbers
}

\author{
by
}

\author{
M. B. Levin (Tel-Aviv) \\ Dedicated to Professor N. M. Korobov \\ on the occasion of his 80th birthday
}

\section{Introduction}

1.1. A number $\alpha \in(0,1)$ is said to be normal to base $q$ if in the $q$-ary expansion of $\alpha, \alpha=. d_{1} d_{2} \ldots\left(d_{i} \in \Delta=\{0,1, \ldots, q-1\}, i=1,2, \ldots\right)$, each fixed finite block of digits of length $k$ appears with an asymptotic frequency of $q^{-k}$ along the sequence $\left(d_{i}\right)_{i \geq 1}$. Normal numbers were introduced by Borel (1909). tity

1.1.1. Let $\left(x_{n}\right)_{n \geq 1}$ be an arbitrary sequence of real numbers. The quan-

(1) $D(N)=D\left(N,\left(x_{n}\right)_{n \geq 1}\right)=\sup _{\gamma \in(0,1]}\left|\#\left\{0 \leq n<N \mid\left\{x_{n}\right\}<\gamma\right\} / N-\gamma\right|$

is called the discrepancy of $\left(x_{n}\right)_{n=1}^{N}$, where $\{x\}=x-[x]$ is the fractional part of $x$. The sequence $\left\{x_{n}\right\}_{n \geq 1}$ is said to be uniformly distributed (u.d.) in $[0,1)$ if $D(N) \rightarrow 0$.

1.1.2. It is known that a number $\alpha$ is normal to base $q$ if and only if the sequence $\left\{\alpha q^{n}\right\}_{n \geq 0}$ is u.d. (Wall, 1949). Borel proved that almost every number (in the sense of Lebesgue measure) is normal to base $q$. In [G], Gal and Gal proved that

$$
D\left(N,\left\{\alpha q^{n}\right\}_{n \geq 0}\right)=O\left(\left(N^{-1} \log \log N\right)^{1 / 2}\right) \quad \text { for a.e. } \alpha .
$$

1.2. In [K1] Korobov posed the problem of finding a function $\psi$ with maximum decay, such that

$$
\exists \alpha: D\left(N,\left\{\alpha q^{n}\right\}_{n \geq 0}\right) \leq \psi(N), \quad N=1,2, \ldots
$$

1991 Mathematics Subject Classification: 11K16, 11K38.

Work supported in part by the Israel Science Foundation Grant No. 0366-172. 
He showed that $\psi(N)=O\left(N^{-1 / 2}\right)$ (see [K1]). The lower bound of the discrepancy for the Champernowne and Davenport-Erdös normal numbers was found by Schiffer $[\mathrm{S}]$ :

$$
D\left(N,\left\{\alpha q^{n}\right\}_{n \geq 1}\right) \geq K / \log N \quad \text { with } K>0, N=2,3, \ldots
$$

For a bibliography on Korobov's problem see [Po, L1].

1.3. In [L2] we proposed using small discrepancy sequences (van der Corput type sequences and $\{n \alpha\}_{n \geq 0}$ ) to construct normal numbers, and announced that

$$
\psi(N)=O\left(N^{-1} \log ^{2} N\right) .
$$

This result is proved below. The estimate of $\psi(N)$ was previously known to be $O\left(N^{-2 / 3} \log ^{4 / 3} N\right)$ (Korobov [K2] for $q$ prime, and Levin [L1] for arbitrary integer $q$ ). We note that the estimate obtained cannot be improved essentially, since according to W. Schmidt, 1972 (see [N, p. 24]), for any sequence of reals,

$$
\varlimsup_{N \rightarrow \infty} N D(N) / \log N>0 .
$$

1.4. Let $x=\left[a_{0}(x) ; a_{1}(x), a_{2}(x), \ldots\right]$ be the continued fraction expansion of $x$, with partial quotients $a_{i}(x)$. For an integer $b$ and $Q>1$ let $\sum a_{i}(b / Q)$ denote the sum of all partial quotients of $b / Q$. Following $[\mathrm{P}]$ we prove (see Lemma 3 ) that there exists an integer sequence $b_{m}$ and a constant $K>0$ with

$$
\sum_{r=1}^{m} \sum a_{i}\left(\left\{b_{m} / q^{r}\right\}\right) \leq K m^{3}, \quad m=1,2, \ldots
$$

\section{TheOREM 1. Let}

$$
\alpha=\sum_{m \geq 1} \frac{1}{q^{n_{m}}} \sum_{0 \leq k<q^{m}}\left\{\frac{b_{m} k}{q^{m}}\right\} \frac{1}{q^{m k}}
$$

where $b_{m}$ satisfy $(2)$,

$$
n_{1}=0 \quad \text { and } \quad n_{k}=\sum_{1 \leq r<k} r q^{r}, \quad k=2,3, \ldots
$$

Then the number $\alpha$ is normal to base $q$, and

$$
D\left(N,\left\{\alpha q^{n}\right\}_{n \geq 0}\right)=O\left(N^{-1} \log ^{3} N\right) .
$$

1.5. Let $\left(p_{i, j}^{\prime}\right)_{i, j \geq 1}$ be Pascal's triangle:

$$
p_{i, 1}^{\prime}=p_{1, i}^{\prime}=1, \quad i=1,2, \ldots, \quad p_{i, j}^{\prime}=p_{i, j-1}^{\prime}+p_{i-1, j}^{\prime}, \quad i, j=2,3, \ldots,
$$

and $\left(p_{i, j}\right)_{i, j \geq 1}$ be Pascal's triangle $\bmod 2$ :

$$
p_{i, j} \equiv p_{i, j}^{\prime} \bmod 2, \quad i, j=1,2, \ldots
$$


Every integer $n \geq 0$ has a unique digit expansion in base $q$,

$$
n=\sum_{j \geq 1} e_{j}(n) q^{j-1} \quad \text { with } e_{j}(n) \in \Delta=\{0, \ldots, q-1\},
$$

$j=1,2, \ldots$, and $e_{j}(n)=0$ for all sufficiently large $j$.

TheOREM 2. Let

$$
\alpha=\sum_{m \geq 1} \frac{1}{q^{n_{m}}} \sum_{0 \leq n<q^{2^{m}}} \frac{1}{q^{n 2^{m}}} \sum_{i=1}^{2^{m}} \frac{d_{i}(n)}{q^{i}}
$$

where

(8) $d_{i}(n) \equiv \sum_{j \geq 1} p_{i, j} e_{j}(n) \bmod q, \quad d_{i}(n) \in \Delta, \quad i=1, \ldots, 2^{m}, n \in\left[0, q^{2^{m}}\right)$,

$$
n_{1}=0 \quad \text { and } \quad n_{m}=\sum_{1 \leq r<m} 2^{r} q^{2^{r}}, \quad m=2,3, \ldots
$$

Then the number $\alpha$ is normal to base $q$ and

$$
D\left(N,\left\{\alpha q^{n}\right\}_{n \geq 0}\right)=O\left(N^{-1} \log ^{2} N\right) .
$$

REMARK 1. We use here the sequence of $2^{m} \times 2^{m}$ matrices of Pascal's triangle $\bmod 2$. A similar result is valid for the sequence of $m \times m$ matrices of Pascal's triangle (or $m \times m$ matrices of Pascal's triangle $\bmod p$ ) but with $D\left(N,\left\{\alpha q^{n}\right\}_{n \geq 0}\right)=O\left(N^{-1} \log ^{3} N\right)$, where $\alpha$ is denoted by a concatenation of blocks $\omega_{m}$ :

$$
\alpha=. \omega_{1} \ldots \omega_{m} \ldots,
$$

where

$$
\omega_{m}=\left(d_{1}(1) \ldots d_{m}(1) \ldots d_{1}\left(q^{m}\right) \ldots d_{m}\left(q^{m}\right)\right), \quad m=1,2, \ldots,
$$

and

$$
d_{i}(n) \equiv \sum_{j \geq 1} p_{i, j} e_{j}(n) \bmod q .
$$

REMARK 2 . Let $\left(\sigma_{i}\right)_{i \geq 1}$ be any sequence of substitutions of the set $\Delta=$ $\{0,1, \ldots, q-1\}$. The proof of Theorem 2 does not change if in (8) we use the functions $\sigma_{i}\left(e_{i}(n)\right)$ instead of the functions $e_{i}(n)$ (see [B], [N, p. 25]).

2. Proof of the theorems. Let $m \geq 1, b, i$ be integers, $0 \leq i<m$, $(b, q)=1$,

$$
\begin{aligned}
& \alpha_{m}=\alpha_{m}(b)=\sum_{0 \leq k<q^{m}}\left\{\frac{b k}{q^{m}}\right\} \frac{1}{q^{m k}}, \\
& \alpha_{m n i}=\left[q^{2 m-i}\left\{\alpha_{m} q^{i+m n}\right\}\right] / q^{2 m-i}
\end{aligned}
$$


It is easy to see that $\left\{\left\{b n / q^{m}\right\} q^{i}\right\}=\left\{b n / q^{m-i}\right\}$, and

$$
\begin{aligned}
\left\{\alpha_{m} q^{i+m n}\right\} & =\left\{\left\{\frac{b n}{q^{m}}\right\} q^{i}+\left\{\frac{b(n+1)}{q^{m}}\right\} \frac{1}{q^{m-i}}+\left\{\frac{b(n+2)}{q^{m}}\right\} \frac{1}{q^{2 m-i}}+\ldots\right\} \\
& =\left\{\frac{b n}{q^{m-i}}\right\}+\left\{\frac{b(n+1)}{q^{m}}\right\} \frac{1}{q^{m-i}}+\left\{\frac{b(n+2)}{q^{m}}\right\} \frac{1}{q^{2 m-i}}+\ldots
\end{aligned}
$$

Therefore

$$
\alpha_{m n i}=\left\{\frac{b n}{q^{m-i}}\right\}+\left\{\frac{b(n+1)}{q^{m}}\right\} \frac{1}{q^{m-i}} .
$$

Let $N \in\left[1, m q^{m}\right]$ be an integer, $\gamma \in(0,1]$,

$$
A\left(\gamma, N,\left(x_{n}\right)\right)= \begin{cases}\#\left\{0 \leq n<N \mid\left\{x_{n}\right\}<\gamma\right\} & \text { for } \gamma>0 \\ 0 & \text { for } \gamma \leq 0\end{cases}
$$

and

$$
A\left(\gamma, Q, P,\left(x_{n}\right)\right)=\#\left\{Q \leq n<Q+P \mid\left\{x_{n}\right\}<\gamma\right\} .
$$

Hence and from (10) we obtain

$$
\begin{aligned}
A\left(\gamma, N,\left\{\alpha_{m} q^{n}\right\}_{n \geq 0}\right)= & A\left(\gamma, m[N / m],\left\{\alpha_{m} q^{n}\right\}_{n \geq 0}\right) \\
& +A\left(\gamma, m[N / m], N-m[N / m],\left\{\alpha_{m} q^{n}\right\}_{n \geq 0}\right) \\
= & \sum_{i=0}^{m-1} A\left(\gamma,[N / m],\left\{\alpha_{m} q^{i+m n}\right\}_{n \geq 0}\right)+\theta m
\end{aligned}
$$

with $\theta \in[0,1]$.

Let $c=\left[q^{m} \gamma\right], N_{1} \in\left[1, q^{m}\right]$ and $0 \leq i<m$. From (11) and (13) we deduce

$$
\begin{aligned}
A\left(\frac{c-1}{q^{m}}, N_{1},\left(\alpha_{m n i}\right)_{n \geq 0}\right) & \leq A\left(\gamma, N_{1},\left\{\alpha_{m} q^{m n+i}\right\}_{n \geq 0}\right) \\
& \leq A\left(\frac{c+1}{q^{m}}, N_{1},\left(\alpha_{m n i}\right)_{n \geq 0}\right) .
\end{aligned}
$$

Lemma 1. Let $N \in\left[1, m q^{m}\right]$ be an integer, $\gamma \in(0,1],(b, q)=1$. Then

$$
\begin{gathered}
A\left(\gamma, N,\left\{\alpha_{m} q^{n}\right\}_{n \geq 0}\right) \\
=\gamma N+\varepsilon_{1}\left(4 m+3 \sum_{i=1}^{m} \max _{1 \leq N \leq q^{i}} N D\left(N,\left\{b n / q^{i}\right\}_{n \geq 0}\right)\right), \\
A\left(\gamma, m q^{m},\left\{\alpha_{m} q^{n}\right\}_{n \geq 0}\right)=\gamma m q^{m}+3 \varepsilon_{2} m
\end{gathered}
$$

with $\left|\varepsilon_{j}\right|<1, j=1,2$. 
Proof. Let $0 \leq i<m, d, d_{1}$, and $d_{2}$ be integers, $d=d_{1} q^{i}+d_{2}, d_{1} \in$ $\left[0, q^{m-i}\right), d_{2} \in\left[0, q^{i}\right)$. By (12) and (13) we get

$$
\begin{aligned}
& A\left(\frac{d}{q^{m}}, N_{1},\left(\alpha_{m n i}\right)_{n \geq 0}\right) \\
& \quad=\#\left\{0 \leq n<N_{1} \mid\left\{\frac{b n}{q^{m-i}}\right\}+\left\{\frac{b(n+1)}{q^{m}}\right\} \frac{1}{q^{m-i}}<\frac{d_{1}}{q^{m-i}}+\frac{1}{q^{m-i}} \cdot \frac{d_{2}}{q^{i}}\right\} .
\end{aligned}
$$

Consequently,

$$
A\left(d / q^{m}, N_{1},\left(\alpha_{m n i}\right)_{n \geq 0}\right)=T_{1}\left(N_{1}\right)+T_{2}\left(N_{1}\right),
$$

where

(20) $T_{1}(N)=\#\left\{0 \leq n<N \mid\left\{\frac{b n}{q^{m-i}}\right\}<\frac{d_{1}}{q^{m-i}}\right\}$,

(21) $T_{2}(N)=\#\left\{0 \leq n<N \mid\left\{\frac{b n}{q^{m-i}}\right\}=\frac{d_{1}}{q^{m-i}}\right.$ and $\left.\left\{\frac{b(n+1)}{q^{m}}\right\}<\frac{d_{2}}{q^{i}}\right\}$.

Let $N_{1}=N_{2} q^{m-i}+N_{3}$ with $N_{3} \in\left[0, q^{m-i}\right)$ and $N_{2} \in\left[0, q^{i}\right)$. It is easy to see that

$$
T_{1}\left(N_{1}\right)=T_{1}\left(q^{m-i} N_{2}\right)+T_{1}\left(N_{3}\right) .
$$

We see from (20) and (1) that

$$
T_{1}\left(N_{2} q^{m-i}\right)=N_{2} d_{1},
$$

and

$$
T_{1}\left(N_{3}\right)=\frac{d_{1}}{q^{m-i}} N_{3}+\varepsilon N_{3} D\left(N_{3},\left\{\frac{b n}{q^{m-i}}\right\}_{n \geq 0}\right) \quad \text { with }|\varepsilon| \leq 1 .
$$

This yields

(23) $\quad T_{1}\left(N_{1}\right)=\frac{d_{1}}{q^{m-i}} N_{1}+\varepsilon \max _{1 \leq N<q^{m-i}} N D\left(N,\left\{\frac{b n}{q^{m-i}}\right\}_{n \geq 0}\right)$ with $|\varepsilon| \leq 1$.

Now we compute $T_{2}(N)$. Let $d_{0}$ be an integer, $d_{0} \equiv d_{1} b^{-1} \bmod q^{m-i}$ with $d_{0} \in\left[0, q^{m-i}\right)$, and

$$
Y=\left\{0 \leq n<N_{1} \mid\left\{b n / q^{m-i}\right\}=d_{1} / q^{m-i}\right\} .
$$

Clearly if $\left\{b n / q^{m-i}\right\}=d_{1} / q^{m-i}$, then $b n \equiv d_{1} \bmod q^{m-i}, n \equiv d_{0} \bmod q^{m-i}$, and

(24) $Y=\left\{d_{0}+r q^{m-i} \mid 0 \leq r<N_{4}\right\} \quad$ with $N_{4}=\left[\frac{N_{1}-d_{0}-1}{q^{m-i}}\right]+1$.

Combining (21) and (1) we obtain 


$$
\begin{aligned}
T_{2}\left(N_{1}\right) & =\#\left\{n \in Y \mid\left\{\frac{b(n+1)}{q^{m}}\right\}<\frac{d_{2}}{q^{i}}\right\} \\
& =\#\left\{0 \leq r<N_{4} \mid\left\{\frac{b\left(d_{0}+1\right)}{q^{m}}+\frac{b r}{q^{i}}\right\}<\frac{d_{2}}{q^{i}}\right\} \\
& =N_{4} \frac{d_{2}}{q^{i}}+\varepsilon_{2} N_{4} D\left(N_{4},\left\{\frac{b n}{q^{i}}+\theta\right\}_{n \geq 0}\right)
\end{aligned}
$$

with $\theta=b\left(d_{0}+1\right) / q^{m},\left|\varepsilon_{2}\right| \leq 1$.

It follows from (1) that for every real $\theta$,

$$
D\left(N,\left\{x_{n}+\theta\right\}_{n \geq 0}\right) \leq 2 D\left(N,\left\{x_{n}\right\}_{n \geq 0}\right) .
$$

By (24) and (25), this yields

(27) $T_{2}\left(N_{1}\right)=\left[\frac{N_{1}+q^{m-i}-d_{0}-1}{q^{m-i}}\right] \frac{d_{2}}{q^{i}}+2 \varepsilon_{2} \max _{1 \leq N \leq q^{i}} N D\left(N,\left\{b n / q^{i}\right\}_{n \geq 0}\right)$

$$
=N_{1} \frac{d_{2}}{q^{m}}+\varepsilon_{3}+2 \varepsilon_{2} \max _{1 \leq N \leq q^{i}} N D\left(N,\left\{b n / q^{i}\right\}_{n \geq 0}\right)
$$

with $\left|\varepsilon_{j}\right| \leq 1, j=2,3$.

If $N_{1}=q^{m}$, then $N_{4}=q^{i}$, and $N_{4} D\left(N_{4},\left\{b n / q^{i}\right\}_{n \geq 0}\right)=1$. Hence and from $(25)$ and $(26)$ we obtain

$$
T_{2}\left(q^{m}\right)=d_{2}+2 \varepsilon_{4} \quad \text { with }\left|\varepsilon_{4}\right| \leq 1 .
$$

Substituting (23) and (27) into (19), we obtain

$$
\begin{aligned}
A\left(d / q^{m}, N_{1},\left(\alpha_{m n i}\right)_{n \geq 0}\right) & \\
= & N_{1} d / q^{m}+\varepsilon_{5}\left(1+\max _{1 \leq N<q^{m-i}} N D\left(N,\left\{b n / q^{m-i}\right\}_{n \geq 0}\right)\right. \\
& \left.+2 \max _{1 \leq N \leq q^{i}} N D\left(N,\left\{b n / q^{i}\right\}_{n \geq 0}\right)\right) \quad \text { with }\left|\varepsilon_{5}\right| \leq 1 .
\end{aligned}
$$

Using (16) and (15) we get

$$
\begin{aligned}
A\left(\gamma, N_{1},\left\{\alpha_{m} q^{m n+i}\right\}_{n \geq 0}\right) & \\
= & \gamma N_{1}+\varepsilon_{6}\left(2+\max _{1 \leq N<q^{m-i}} N D\left(N,\left\{b n / q^{m-i}\right\}_{n \geq 0}\right)\right. \\
& \left.+2 \max _{1 \leq N \leq q^{i}} N D\left(N,\left\{b n / q^{i}\right\}_{n \geq 0}\right)\right) \quad \text { with }\left|\varepsilon_{6}\right| \leq 1,
\end{aligned}
$$

and

$$
\begin{aligned}
A\left(\gamma, N,\left\{\alpha_{m} q^{n}\right\}_{n \geq 0}\right)= & \theta m+\sum_{i=1}^{m} \gamma[N / m] \\
& +\varepsilon_{7}\left(2 m+3 \sum_{i=1}^{m} \max _{1 \leq N \leq q^{i}} N D\left(N,\left\{b n / q^{i}\right\}_{n \geq 0}\right)\right) \\
= & \gamma N+\varepsilon_{8}\left(4 m+3 \sum_{i=1}^{m} \max _{1 \leq N \leq q^{i}} N D\left(N,\left\{b n / q^{i}\right\}_{n \geq 0}\right)\right),
\end{aligned}
$$


where $\left|\varepsilon_{j}\right| \leq 1, j=7,8$. Assertion (17) is proved. Assertion (18) follows analogously from (22) and (28).

Lemma 2. Let $j \geq 1,1 \leq N \leq q^{j},(b, q)=1$, and $a_{i}(x)$ be partial quotients of $\{x\}$. Then

$$
N D\left(N,\left\{b n / q^{j}\right\}_{n \geq 0}\right) \leq \sum a_{i}\left(b / q^{j}\right) .
$$

For the proof of this well-known theorem, see for example [N, p. 26].

LEmma 3. There exists a constant $K>0$ and integers $c_{m} \in\left[0, q^{m}\right)$ such that

$$
\sum_{r=1}^{m} \sum a_{i}\left(\left\{c_{m} / q^{r}\right\}\right) \leq K m^{3}, \quad m=1,2, \ldots
$$

Pro of. According to [P, p. 2144] there exist constants $K_{q}$ such that

$$
\sum_{1 \leq c \leq q^{r},(c, q)=1} \sum a_{i}\left(c / q^{r}\right) \leq K_{q} q^{r} r^{2}, \quad r=1,2, \ldots
$$

Therefore

$$
\begin{aligned}
& \sum_{1 \leq c \leq q^{m},(c, q)=1} \sum_{r=1}^{m} \sum a_{i}\left(\left\{c / q^{r}\right\}\right) \\
= & \sum_{r=1}^{m} q^{m-r} \sum_{1 \leq c \leq q^{r},(c, q)=1} \sum a_{i}\left(c / q^{r}\right) \leq \sum_{r=1}^{m} q^{m} K_{q} r^{2} \leq K_{q} q^{m} m^{3} .
\end{aligned}
$$

Let $\phi\left(q^{m}\right)=\#\left\{1 \leq c \leq q^{m} \mid(c, q)=1\right\}$ and $K=K_{q} q / \phi(q)$. It is known that $\phi\left(q^{m}\right)=q^{m-1} \phi(q)$. Now the assertion of Lemma 3 follows from (29).

Corollary. Let $1 \leq N \leq m q^{m}$. Then

$$
A\left(\gamma, N,\left\{\alpha_{m}\left(b_{m}\right) q^{n}\right\}_{n \geq 0}\right)=\gamma N+O\left(m^{3}\right) .
$$

The statement follows from (1), (2), (10), and Lemmas 1-3.

Applying (3) and (10) we get

$\left\{\alpha q^{n_{m}+n}\right\}=\left\{\alpha_{m}\left(b_{m}\right) q^{n}\right\}+\theta q^{n-m q^{m}} \quad$ with $0<\theta<1$ and $0 \leq n<m q^{m}$.

Hence and from (13) we have, for $N \in\left[1, m q^{m}\right]$,

$$
\begin{aligned}
A\left(\gamma-1 / q^{m}, N-m,\left\{\alpha_{m}\left(b_{m}\right) q^{n}\right\}_{n \geq 0}\right) & \leq A\left(\gamma, N,\left\{\alpha q^{n_{m}+n}\right\}_{n \geq 0}\right) \\
& \leq A\left(\gamma, N,\left\{\alpha_{m}\left(b_{m}\right) q^{n}\right\}_{n \geq 0}\right) .
\end{aligned}
$$

By using (30) and (14), we obtain

$$
A\left(\gamma, n_{m}, N,\left\{\alpha q^{n}\right\}_{n \geq 0}\right)=\gamma N+O\left(m^{3}\right) \quad \text { with } 1 \leq N \leq m q^{m} .
$$

Similarly, from (18) we deduce that

$$
A\left(\gamma, n_{m}, m q^{m},\left\{\alpha q^{n}\right\}_{n \geq 0}\right)=\gamma m q^{m}+O(m) .
$$


End of the proof of Theorem 1. For every $N \geq 1$ there exists an integer $k$ such that $N \in\left[n_{k}, n_{k+1}\right)$. By (4) this yields

(33) $N=n_{k}+R \quad$ with $0 \leq R<k q^{k}, N>(k-1) q^{k-1}, k \leq 2 \log _{q} N$.

Applying (4), (14) and (31)-(33) we obtain

$$
\begin{aligned}
A\left(\gamma, N,\left\{\alpha q^{n}\right\}_{n \geq 0}\right) & =\sum_{r=1}^{k-1} A\left(\gamma, n_{r}, r q^{r},\left\{\alpha q^{n}\right\}_{n \geq 0}\right)+A\left(\gamma, n_{k}, R,\left\{\alpha q^{n}\right\}_{n \geq 0}\right) \\
& =\sum_{r=1}^{k-1}\left(\gamma r q^{r}+O(r)\right)+\gamma R+O\left(k^{3}\right) \\
& =\gamma N+O\left(k^{3}\right)=\gamma N+O\left(\log ^{3} N\right) .
\end{aligned}
$$

Thus, by (1), the theorem is proved.

Proof of Theorem 2. In [So] Sobol' proposed the use of Pascal's triangle mod 2 to construct small discrepancy sequences (see also [F], [N]). Here we use Pascal's triangle mod 2 to construct normal numbers.

Let $P_{n}$ be a sequence of a $2^{n} \times 2^{n}$ matrices such that

$$
P_{1}=\left(\begin{array}{ll}
1 & 1 \\
1 & 0
\end{array}\right), \quad \ldots, \quad P_{n+1}=\left(\begin{array}{cc}
P_{n} & P_{n} \\
P_{n} & 0
\end{array}\right),
$$

It is easy to prove by induction that $P_{n}$ is the $2^{n} \times 2^{n}$ upper left-hand corner of Pascal's triangle (5), and $P_{n}$ is a triangular-type matrix. The following lemma is proved in $[\mathrm{BH}]$ for Pascal's triangle, and it is clearly valid also for Pascal's triangle mod 2.

Lemma 4. The determinant of any $n \times n$ array taken with its first row along a row of ones, or with its first column along a column of ones in Pascal's triangle, written in rectangular form, is one.

From (7) we have

$$
\left\{\alpha q^{n_{m}+2^{m} n+k}\right\}=. d_{k+1}(n) d_{k+2}(n) \ldots d_{2^{m}}(n) d_{1}(n+1) \ldots
$$

Let $1 \leq k, i \leq 2^{m}$ and

$$
\alpha_{k i}(n)=\left[\left\{\alpha q^{n_{m}+2^{m} n+k}\right\} q^{i}\right] / q^{i} .
$$

It is easy to see that

$$
\begin{aligned}
& \alpha_{k i}(n) \\
& = \begin{cases}\cdot d_{k+1}(n) \ldots d_{k+i}(n) & \text { if } k+i \leq 2^{m}, \\
. d_{k+1}(n) \ldots d_{2^{m}}(n) d_{1}(n+1) \ldots d_{k+i-2^{m}}(n+1) & \text { otherwise. }\end{cases}
\end{aligned}
$$

Lemma 5. Let $m, k, i, B, f$ be integers, $1 \leq i, k \leq 2^{m}, B \in\left[0, q^{2^{m}-i}\right)$, $f \in\left[0, q^{i}\right)$. Then

$$
A\left(f / q^{i}, B q^{i}, q^{i},\left(\alpha_{k i}(n)\right)_{n \geq 0}\right)=f+2 \varepsilon \quad \text { with }|\varepsilon|<1 .
$$


Proof. CASE 1. Let $k+i \leq 2^{m}, c_{j} \in \Delta=\{0,1, \ldots, q-1\}(j=1, \ldots, i)$. We examine the system of equations

$$
d_{k+j}(n)=c_{j}, \quad j=1, \ldots, i, n \in\left[B q^{i},(B+1) q^{i}\right) .
$$

According to (8) this system is equivalent to the system of $i$ congruences

$$
\sum_{1 \leq \nu \leq 2^{m}} p_{k+j, \nu} e_{\nu}\left(n+B q^{i}\right) \equiv c_{j} \bmod q, \quad j=1, \ldots, i, n \in\left[0, q^{i}\right) .
$$

Applying (6) we see that $e_{\nu}\left(n+B q^{i}\right)=e_{\nu}(n)+e_{\nu}\left(B q^{i}\right), \nu=1,2, \ldots$, and

$$
\begin{aligned}
\sum_{1 \leq \nu \leq i} p_{k+j, \nu} e_{\nu}(n) & \\
& \equiv c_{j}-\sum_{i<\nu \leq 2^{m}} p_{k+j, \nu} e_{\nu}\left(B q^{i}\right) \bmod q, \quad j=1, \ldots, i,
\end{aligned}
$$

with $n \in\left[0, q^{i}\right)$. It follows from Lemma 4 that

$$
\left|\operatorname{det}\left(p_{k+j, \nu}\right)_{1 \leq j, \nu \leq i}\right|=1 .
$$

For any $c_{1}, \ldots, c_{i}$ the system (38) has a unique solution $\left(e_{1}(n), \ldots, e_{i}(n)\right)$, and consequently there exists a unique $n_{0} \in\left[B q^{i},(B+1) q^{i}\right)$ satisfying (37).

From (36) and (37) we see that the set $\left\{\alpha_{k i}(n) \mid n \in\left[B q^{i},(B+1) q^{i}\right)\right\}$ coincides with $\left\{j / q^{i} \mid j \in\left[0, q^{i}\right)\right\}$. Hence and from (14) we have

$$
A\left(f / q^{i}, B q^{i}, q^{i},\left(\alpha_{k i}(n)\right)_{n \geq 0}\right)=f .
$$

CASE 2. Let $k+i>2^{m}, l_{1}=2^{m}-k$. As in (37) and (38), the system of equations

$$
\begin{aligned}
d_{k+j}(n) & =c_{j}, & & j=1, \ldots, l_{1}, \\
d_{j}(n+1) & =c_{j+l_{1}}, & j & =1, \ldots, i-l_{1},
\end{aligned}
$$

with $n \in\left[B q^{i},(B+1) q^{i}\right)$, is equivalent to the systems of congruences

$$
\begin{aligned}
& \sum_{1 \leq \nu \leq i} p_{k+j, \nu} e_{\nu}(n) \\
& \quad \equiv c_{j}-\sum_{i<\nu \leq 2^{m}} p_{k+j, \nu} e_{\nu}\left(B q^{i}\right) \bmod q, \quad j=1, \ldots, l_{1}, \\
& \sum_{1 \leq \nu \leq i} p_{j, \nu} e_{\nu}(n+1) \\
& \equiv c_{j+l_{1}}-\sum_{i<\nu \leq 2^{m}+1} p_{j, \nu} e_{\nu}\left(\left(B+\left[(n+1) / q^{i}\right]\right) q^{i}\right) \bmod q,
\end{aligned}
$$

where $j=1, \ldots, i-l_{1}$ and $n \in\left[0, q^{i}\right)$.

Let $n=n_{1}+n_{2} q^{l_{1}}$ with $n_{1} \in\left[0, q^{l_{1}}\right)$ and $n_{2} \in\left[0, q^{i-l_{1}}\right)$. It is evident that $e_{\nu}(n)=e_{\nu}\left(n_{1}\right)$ for $\nu=1, \ldots, l_{1}$.

The matrix $P_{m}$ is triangular. Hence

$$
p_{k+j, \nu}=0 \quad \text { with } \nu>2^{m}-k-j=l_{1}-j .
$$

The system (43) is equivalent to the following system of congruences: 


$$
\begin{aligned}
\sum_{1 \leq \nu \leq l_{1}} p_{k+j, \nu} e_{\nu}\left(n_{1}\right) & \\
& \equiv c_{j}-\sum_{i<\nu \leq 2^{m}} p_{k+j, \nu} e_{\nu}\left(B q^{i}\right) \bmod q, \quad j=1, \ldots, l_{1},
\end{aligned}
$$

where $n_{1} \in\left[0, q^{l_{1}}\right)$ and $n_{2} \in\left[0, q^{i-l_{1}}\right)$.

Applying (39) with $i=l_{1}$ shows that this system has a unique solution with $\left(e_{1}\left(n_{1}\right), \ldots, e_{l_{1}}\left(n_{1}\right)\right)$. Consequently, there exists a unique solution $n_{1}=$ $n_{1}^{\prime} \in\left[0, q^{l_{1}}\right)$ satisfying (45).

By (41) and (43) we obtain

$$
\begin{array}{r}
\left\{\left(d_{k+1}\left(n_{1}+n_{2} q^{l_{1}}+B q^{i}\right), \ldots, d_{k+l_{1}}\left(n_{1}+n_{2} q^{l_{1}}+B q^{i}\right)\right) \mid 0 \leq n_{1}<q^{l_{1}}\right\} \\
=\left\{\left(c_{1}, \ldots, c_{l_{1}}\right) \mid c_{j} \in \Delta, j=1, \ldots, l_{1}\right\} .
\end{array}
$$

Now we examine the system (44) with $n_{1}=n_{1}^{\prime}$ the solution of (45).

CASE 2.1. Let $n_{1}^{\prime} \leq q^{l_{1}}-2$. Bearing in mind that

$$
e_{\nu}(n+1)=e_{\nu}\left(n_{1}^{\prime}+1+q^{l_{1}} n_{2}\right)=e_{\nu}\left(n_{1}^{\prime}+1\right)+e_{\nu}\left(q^{l_{1}} n_{2}\right),
$$

we deduce from (44) that

$$
\begin{aligned}
& \sum_{l_{1}<\nu \leq i} p_{j, \nu} e_{\nu}\left(q^{l_{1}} n_{2}\right) \\
& \quad \equiv c_{j+l_{1}}-\sum_{1 \leq \nu \leq l_{1}} p_{j, \nu} e_{\nu}\left(n_{1}^{\prime}+1\right)-\sum_{i<\nu \leq 2^{m}} p_{j, \nu} e_{\nu}\left(B q^{i}\right) \bmod q
\end{aligned}
$$

with $j=1, \ldots, i-l_{1}$ and $0 \leq n_{2}<q^{i-l_{1}}$.

Applying Lemma 4 we obtain a unique solution for this system with $\left(e_{l_{1}+1}\left(q^{l_{1}} n_{2}\right), \ldots, e_{i}\left(q^{l_{1}} n_{2}\right)\right)$.

By (42) and (44) we get

$$
\begin{aligned}
& \left\{\left(d_{1}\left(n_{1}^{\prime}+n_{2} q^{l_{1}}+B q^{i}+1\right), \ldots, d_{i-l_{1}}\left(n_{1}^{\prime}+n_{2} q^{l_{1}}+B q^{i}+1\right)\right) \mid\right. \\
& \left.\quad 0 \leq n_{2}<q^{i-l_{1}}\right\}=\left\{\left(c_{l_{1}+1}, \ldots, c_{i}\right) \mid c_{l_{1}+j} \in \Delta, j=1, \ldots, i-l_{1}\right\} .
\end{aligned}
$$

Let

$$
\begin{aligned}
& F=\left\{d_{k+1}(n) \ldots d_{2^{m}}(n) d_{1}(n+1) \ldots d_{k+i-2^{m}}(n+1) \mid\right. \\
& \left.\quad 0 \leq n_{1}<q^{l_{1}}-1,0 \leq n_{2}<q^{i-l_{1}}, n=n_{1}+n_{2} q^{l_{1}}+B q^{i}\right\},
\end{aligned}
$$

and

$$
g_{\nu}=d_{k+\nu}\left(q^{l_{1}}-1+B q^{i}\right), \quad \nu=1, \ldots, l_{1} .
$$

From (46) and (47) we have

(50) $F=\left\{\left(c_{1}, \ldots, c_{i}\right) \mid c_{j} \in \Delta, j=1, \ldots, i,\left(c_{1}, \ldots, c_{l_{1}}\right) \neq\left(g_{1}, \ldots, g_{l_{1}}\right)\right\}$

and

$$
\# F=q^{i}-q^{i-l_{1}} .
$$

CASE 2.2. Let $n_{1}^{\prime}=q^{l_{1}}-1, n_{2} \in\left[0, q^{i-l_{1}}-2\right]$ and $n=n_{1}^{\prime}+n_{2} q^{l_{1}}$. Then $e_{\nu}\left(n_{1}^{\prime}+1\right)=0$ for $1 \leq \nu \leq l_{1}$ and $e_{\nu}(n+1)=e_{\nu}\left(\left(n_{2}+1\right) q^{l_{1}}\right)$ for $l_{1}<\nu \leq i$. 
The system (44) is equivalent to the following system of congruences:

$$
\begin{aligned}
& \sum_{l_{1}<\nu \leq i} p_{j, \nu} e_{\nu}\left(\left(n_{2}+1\right) q^{l_{1}}\right) \\
& \quad \equiv c_{j+l_{1}}-\sum_{i<\nu \leq 2^{m}} p_{j, \nu} e_{\nu}\left(B q^{i}\right) \bmod q, \quad j=1, \ldots, i-l_{1},
\end{aligned}
$$

with $0 \leq n_{2} \leq q^{i-l_{1}}-2$.

For $n_{2} \in\left[0, q^{i-l_{1}}-2\right]$ we have the $q^{i-l_{1}}-1$ distinct vectors of

$$
\left(e_{l_{1}+1}\left(\left(n_{2}+1\right) q^{l_{1}}\right), \ldots, e_{i}\left(\left(n_{2}+1\right) q^{l_{1}}\right)\right) .
$$

Using Lemma 4 and by (52) we obtain for $n_{2} \in\left[0, q^{i-l_{1}}-2\right]$ the $q^{i-l_{1}}-1$ distinct vectors of $\left(c_{l_{1}+1}, \ldots, c_{i}\right)$.

Let

$$
\begin{array}{r}
G=\left\{\left(g_{1}, \ldots, g_{l_{1}}, d_{1}\left(\left(n_{2}+1\right) q^{l_{1}}+B q^{i}\right), \ldots, d_{i-l_{1}}\left(\left(n_{2}+1\right) q^{l_{1}}+B q^{i}\right)\right) \mid\right. \\
\left.0 \leq n_{2} \leq q^{i-l_{1}}-2\right\} .
\end{array}
$$

From (42), (44) and (52) we find that \#G= $q^{i-l_{1}}-1$, and from (46) and (48)-(51) that $\#(F \cup G)=q^{i}-1$. Hence and from (36) the set $\left\{\alpha_{k i}(n) \mid\right.$ $\left.n \in\left[B q^{i},(B+1) q^{i}-2\right]\right\}$ coincides with $q^{i}-1$ distinct values of $j / q^{i}$ with $j \in\left[0, q^{i}\right)$. By (14) we get

$$
A\left(f / q^{i}, B q^{i}, q^{i},\left(\alpha_{k i}(n)\right)_{n \geq 0}\right)=f+2 \varepsilon \quad \text { with }|\varepsilon|<1 .
$$

Hence and from (40) we have the assertion of Lemma 5.

\section{COROllary 1.}

$$
A\left(\gamma, B q^{i}, q^{i},\left\{\alpha q^{n_{m}+2^{m} n+k}\right\}_{n \geq 0}\right)=\gamma q^{i}+4 \varepsilon \quad \text { with }|\varepsilon|<1 .
$$

Proof. Analogously to (16), from (14) and (35) we have

$$
\begin{aligned}
A\left(\frac{f-1}{q^{i}}, B q^{i}, q^{i},\left(\alpha_{k i}(n)\right)_{n \geq 0}\right) & \leq A\left(\gamma, B q^{i}, q^{i},\left\{\alpha q^{n_{m}+2^{m} n+k}\right\}_{n \geq 0}\right) \\
& \leq A\left((f+1) / q^{i}, B q^{i}, q^{i},\left(\alpha_{k i}(n)\right)_{n \geq 0}\right)
\end{aligned}
$$

with $f=\left[\gamma q^{i}\right]$. By using Lemma 5 we obtain (53).

Corollary 2. Let $1 \leq N<2^{m} q^{2^{m}}$. Then

(55) $A\left(\gamma, n_{m}, 2^{m} q^{2^{m}},\left\{\alpha q^{n}\right\}_{n \geq 0}\right)=\gamma 2^{m} q^{2^{m}}+5 \varepsilon 2^{m}$

with $|\varepsilon|<1$,

with $|\varepsilon|<1$.

Proof. Let $N^{\prime}=\left[N / 2^{m}\right], N^{\prime \prime}=N-2^{m} N^{\prime}, N^{\prime}=\sum_{i=0}^{2^{m}-1} b_{i} q^{i}$ with $b_{i} \in \Delta$,

(56) $\quad N_{0}=0, \quad N_{j}=\sum_{i=0}^{j-1} b_{2^{m}-i} q^{2^{m}-i}, \quad j=1,2, \ldots, \quad B_{i}=N_{2^{m}-i-1} / q^{i}$. 
It is evident that $B_{i}(i=1,2, \ldots)$ are integers, and $N^{\prime \prime} \in\left[0,2^{m}\right)$. As in (15) we see from (14) that

$$
A\left(\gamma, n_{m}, N,\left\{\alpha q^{n}\right\}_{n \geq 0}\right)=\varepsilon N_{2}+\sum_{k=1}^{2^{m}} A\left(\gamma, N^{\prime},\left\{\alpha q^{n_{m}+2^{m} n+k}\right\}_{n \geq 0}\right),
$$

and

$$
\begin{aligned}
A\left(\gamma, N^{\prime},\left\{\alpha q^{n_{m}+2^{m} n+k}\right\}_{n \geq 0}\right) & \\
& =\sum_{i=1}^{2^{m}} A\left(\gamma, N_{i-1}, b_{2^{m}-i} q^{2^{m}-i},\left\{\alpha q^{n_{m}+2^{m} n+k}\right\}_{n \geq 0}\right) \\
& =\sum_{i=0}^{2^{m}-1} A\left(\gamma, N_{2^{m}-i-1}, b_{i} q^{i},\left\{\alpha q^{n_{m}+2^{m} n+k}\right\}_{n \geq 0}\right) \\
& =\sum_{i=0}^{2^{m}-1} \sum_{B=0}^{b_{i}-1} A\left(\gamma, N_{2^{m}-i-1}+B q^{i}, q^{i},\left\{\alpha q^{n_{m}+2^{m} n+k}\right\}_{n \geq 0}\right) .
\end{aligned}
$$

Using (56) we have

$$
\begin{aligned}
& A\left(\gamma, n_{m}, N,\left\{\alpha q^{n}\right\}_{n \geq 0}\right) \\
& =\varepsilon 2^{m}+\sum_{k=1}^{2^{m}} \sum_{i=0}^{2^{m}} \sum_{B=0}^{b_{i}-1} A\left(\gamma,\left(B_{i}+B\right) q^{i}, q^{i},\left\{\alpha q^{n_{m}+2^{m} n+k}\right\}_{n \geq 0}\right) .
\end{aligned}
$$

Applying (53) we obtain

$$
A\left(\gamma, n_{m}, N,\left\{\alpha q^{n}\right\}_{n \geq 0}\right)=\varepsilon 2^{m}+\sum_{k=1}^{2^{m}} \sum_{i=0}^{2^{m}} \sum_{B=0}^{b_{i}-1}\left(\gamma q^{i}+4 \varepsilon_{i}\right)=\gamma N+5 q \varepsilon_{1} 2^{2 m}
$$

with $\left|\varepsilon_{1}\right| \leq 1$.

Assertion (54) is proved. We prove (55) analogously.

End of the proof of Theorem 2. For every $N \geq q$ there exists an integer $k$ such that $N \in\left[n_{k}, n_{k+1}\right)$. By (9), this yields $N=n_{k}+R$ with $0 \leq R<2^{k} q^{2^{k}}$, $N \geq 2^{(k-1)} q^{2^{k-1}}, 2^{k} \leq 2 \log _{q} N$. Applying (9), (13), (14), (54) and (55) we obtain

$$
\begin{aligned}
A\left(\gamma, N,\left\{\alpha q^{n}\right\}_{n \geq 0}\right)= & \sum_{m=1}^{k-1} A\left(\gamma, n_{m}, 2^{m} q^{2^{m}},\left\{\alpha q^{n}\right\}_{n \geq 0}\right) \\
& +A\left(\gamma, n_{k}, R,\left\{\alpha q^{n}\right\}_{n \geq 0}\right) \\
= & \sum_{m=1}^{k-1}\left(\gamma 2^{m} q^{2^{m}}+O\left(2^{m}\right)\right)+\gamma R+O\left(2^{2 k}\right) \\
= & \gamma N+O\left(2^{2 k}\right)=\gamma N+O\left(\log ^{2} N\right) .
\end{aligned}
$$

Thus, by (1), the theorem is proved. 
Acknowledgments. I am very grateful to Professor Meir Smorodinsky for his hospitality, and to the referee for his valuable suggestions.

\section{References}

[B] R. Bejian, Sur certaines suites présentant une faible discrépance à l'origine, C. R. Acad. Sci. Paris Sér. A 286 (1978), 135-138.

[BH] M. Bicknell and V. E. Hoggart, Jr., Unit determinants in generalized Pascal triangles, Fibonacci Quart. 11 (1978), 131-144.

[F] H. Faure, Discrépance de suites associées à un système de numération (en dimension s), Acta Arith. 41 (1982), 337-351.

[G] I. S. Gal and L. Gal, The discrepancy of the sequence $\left(2^{n} x\right)$, Indag. Math. 26 (1964), 129-143.

[K1] N. M. Korobov, Numbers with bounded quotient and their applications to questions of Diophantine approximation, Izv. Akad. Nauk SSSR Ser. Mat. 19 (1955), 361-380.

[K2] -, Distribution of fractional parts of exponential function, Vestnik Moskov. Univ. Ser. I Mat. Mekh. 21 (1966), no. 4, 42-46.

[L1] M. B. Levin, The distribution of fractional parts of the exponential function, Soviet Math. (Izv. VUZ) 21 (1977), no. 11, 41-47.

[L2] - On the upper bounds of discrepancy of completely uniform distributed and normal sequences, AMS-IMU joint meeting, Jerusalem, Israel, May 24-26, 1995, Abstracts Amer. Math. Soc. 16 (1995), 556-557.

[N] H. Niederreiter, Random Number Generation and Quasi-Monte Carlo Methods, CBMS-NSF Regional Conf. Ser. in Appl. Math. 63, Philadelphia, 1992.

[P] V. N. Popov, Asymptotic formula for the sum of sums of the elements of the continued fractions for numbers of the form a/p, J. Soviet Math. 17 (1981), 21372147.

[Po] A. G. Postnikov, Arithmetic modeling of random processes, Proc. Steklov Inst. Math. 57 (1960).

[S] J. Schiffer, Discrepancy of normal numbers, Acta Arith. 47 (1986), 175-186.

[So] I. M. Sobol', Multidimensional Quadrature Formulas and Haar Functions, Nauka, Moscow, 1969 (in Russian).

School of Mathematical Sciences

Tel-Aviv University

69978 Tel-Aviv, Israel

E-mail:mlevin@math.tau.ac.il

Received on 30.12.1996

and in revised form on 29.5.1998 